\title{
SILACYCLIC DERIVATIVES OF HETEROAROMATIC SULFIDES AS SELECTIVE CHOLESTEROL LEVEL LOWERING AND VASODILATING AGENTS
}

\author{
Edgars Abele, Kira Rubina*, Ramona Abele, Olegs Dzenitis, Pavel Arsenyan, \\ Juris Popelis, Maris Veveris, Dainuvite Meirena, and Edmunds Lukevics
}

Latvian Institute of Organic Synthesis, 21 Aizkraukles street, Riga, LV-1006, Latvia < kira@osi.lv>

\begin{abstract}
Silacyclic derivatives of heteroaromatic sulfides have been prepared by using phase transfer catalytic (PTC) system thiol / silacyclopropyl iodide / solid $\mathrm{K}_{2} \mathrm{CO}_{3}$ / 18-crown-6 / toluene. The target sulfides were isolated in yields up to $70 \%$. The S-derivatives of $\mathrm{N}$-methylimidazolyl, benzoxazolyl and 1,3,4-triazolyl thiols selectively lowered the low density lipoprotein (LDL) level in mice with the high cholesterol diet in nutrition.
\end{abstract}

\section{INTRODUCTION}

Recently the activity of a wide spectrum of heteroaromatic sulfides on the heart and blood circulatory system was described [1]. For example, quinoline sulfides exhibit antihypertensive [2-4], vasodilating [2-3], hypotensive [5, 6], euglicemic and hypolipidemic [7], antithrombotic [8], cardiotonic [9], cardiovascular [1012] and cholesterol- [13] and blood sugar lowering [14] activities and may be used in the treatment of diabetes [15-17] and arthritis [18]. Thiazole and benzothiazole sulfides display vasodilating [19], hypotensive [20] and antihyperglycemic and antihyperlipidemic [21] activities. Imidazole and benzimidazole sulfides exhibit vasodilating [22], antihypertensive [23], hypoglycemic [24], antithrombotic [25], cardiotonic [26, 27], cardiovascular [28], cholesterol [29-31] and blood sugar lowering [32], antidiabetic [33, 34] activities, increase high density lipoprotein cholesterol over lipid fractions [35] and were used in the treatment of atherosclerosis [36-39] and osteoarthritis [40]. Triazole sulfides were used in the treatment of high blood pressure and heart failure [41] and display platelet activator and antithrombotic [42] activities. Oxazole sulfides exhibit an antihypertensive [43] activity. Indole sulfur derivatives were used in the treatment of atherosclerosis [44], arthritis [45, 46], diabetic complications [47] and congestive hearth failure [48].

In our previous work the cholesterol level lowering and vasodilating activities of silicon and germanium containing aliphatic derivatives of heteroaromatic sulfides were studied [1]. The 1methylimidazole, benzothiazole and 2-quinoline derivatives exhibited the highest level of activity. The compounds containing dimethyl $(\beta$-triethylgermylethyl)silylmethyl and $\operatorname{dimethyl}(\beta$ triphenylsilylethyl)silylmethyl substituents were the most active in mice with the high cholesterol diet in nutrition. It was also shown that aliphatic silyl and germyl 1-methylimidazole derivatives possess a considerable vasodilating activity.

In continuation of our investigations in the field of cholesterol lowering agents the silacyclic Sderivatives of $\mathrm{N}-, \mathrm{O}$ - and S-heterocycles have been synthesized under PTC conditions for the purpose to increase the heterocyclic sulfides lipophility and selectivity of action on the high and low density lipoproteins.

\section{MATERIALS AND METHODS \\ CHEMISTRY}

${ }^{1} \mathrm{H}$ NMR spectra were recorded on a Varian 200 Mercury instrument $(200 \mathrm{MHz})$ using $\mathrm{CDCl}_{3}$ as solvent and hexamethyldisiloxane (HMDSO) as internal standard. Mass spectra were registered on a GC-MS HP $6890(70 \mathrm{eV})$. GC analysis was performed on a Chrom-5 instrument equipped with a flame-ionization detector using glass column packed with $5 \%$ OV-101/ Chromosorb W-HP (80-100 mesh) (1.2 m x $3 \mathrm{~mm})$. 1-(3-Iodopropyl)-1-methylsilacyclopentane and 1-(3-iodopropyl)-1-methylsilacyclohexane were obtained by Grignard reaction $[49,50]$.

SYNTHESIS OF SILACYCLIC DERIVATIVES OF HETARYL THIOLS. GENERAL PROCEDURE.

Finely powdered dry $\mathrm{K}_{2} \mathrm{CO}_{3}$ was added to a mixture of $10 \mathrm{mmol}$ of thiol $(1-7), 10 \mathrm{mmol}$ of 1-(3iodopropyl)-1-methylsilacyclopentane or 1-(3-iodopropyl)-1-methylsilacyclohexane and 18-crown-6 (1mmol, $264 \mathrm{mg}$ ) in $25 \mathrm{ml}$ of toluene. The mixture was refluxed with stirring to achieve the disappearance of the substrates, filtered over the thin silica gel layer and concentrated under reduced pressure. The residue was purified by column chromatography using benzene-hexane or benzene-ethyl acetate as eluent.

\section{PHARMACOLOGY}

Cholesterol level lowering and vasodilating activities and the acute toxicity of synthesized compounds were determined as described in Ref. 1 . 


\section{RESULTS AND DISCUSSION}

CHEMISTRY

A simple method for the preparation of silacyclic derivatives of the N-, O- and S-heterocyclic thiols was developed. The phase transfer catalytical system solid $\mathrm{K}_{2} \mathrm{CO}_{3} / 18$-crown-6/toluene was used (Figure 1). The use of the stronger base $(\mathrm{KOH})$ led to the destruction of the alkylating agents. The aimed substances were obtained in good chemical yields (up to 70\%) in a short time under mild conditions (Table 1).

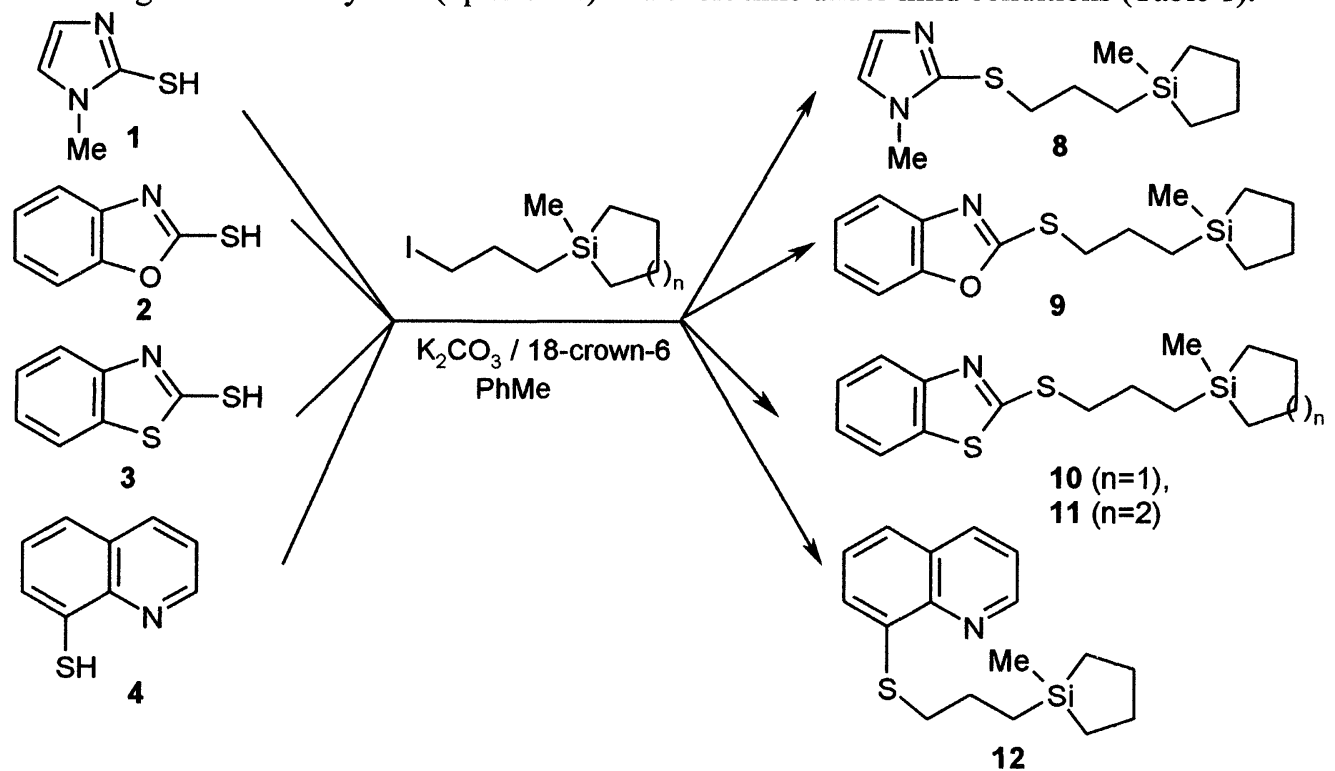

Figure 1.

The alkylation of sulfides $5-7$ simultaneously containing two $\mathrm{SH}$ and $\mathrm{NH}$ targets of reaction led to the S-, N-disubstituted derivatives 13 - 15 (Figure 2).

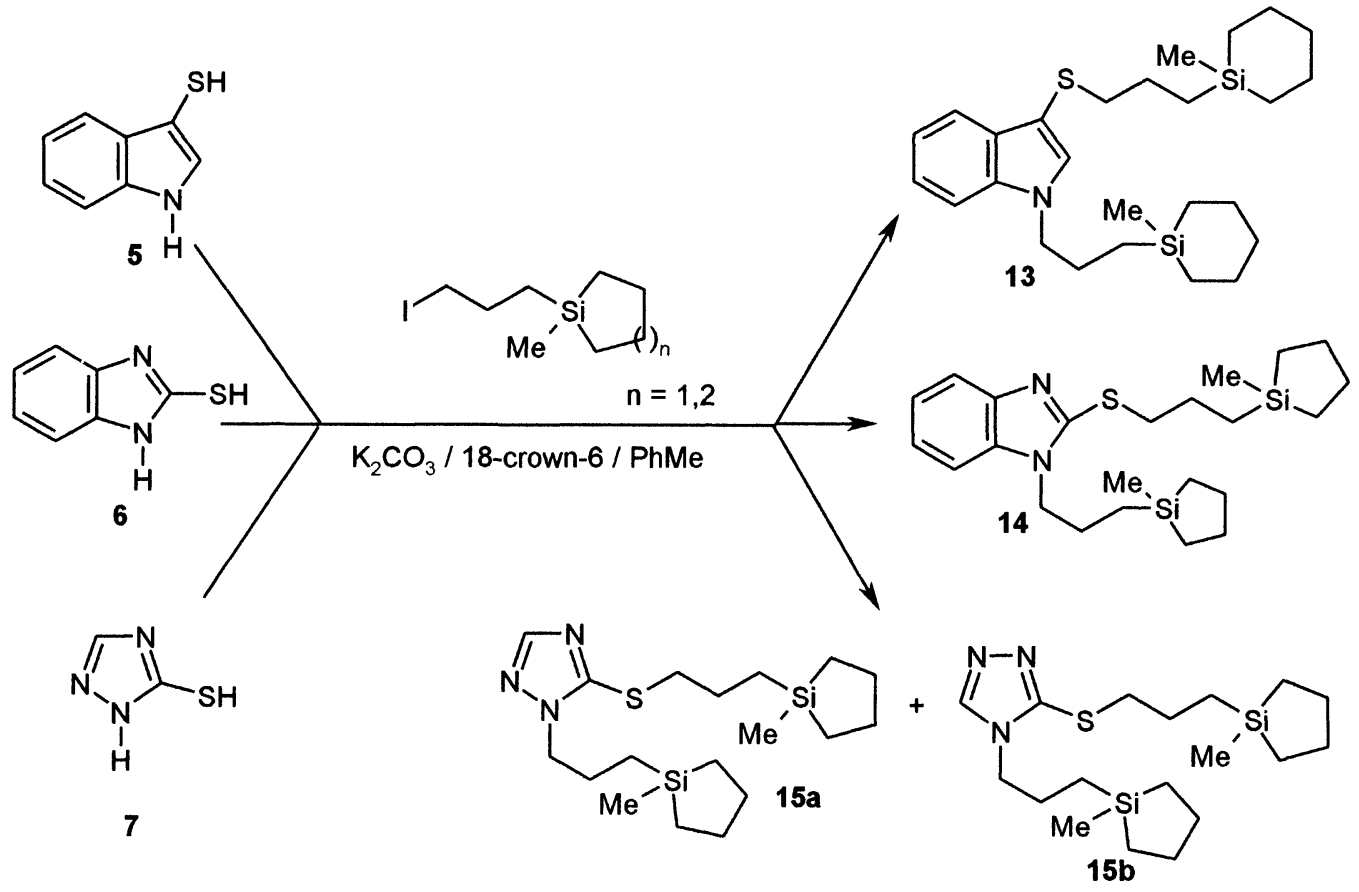

Figure 2.

The 1,2,4-triazole thiol isomerised during the alkylation reaction. Two products have been isolated: 1[3-(1-methyl-1-silacyclopentyl)propyl]-5-\{[3-(1-methyl-1-silacyclopentyl)propyl]thio $\}$-1,2,4-triazole (15a) in $32 \%$ and 3-\{[3-(1-methyl-1-silacyclopentyl)propyl]thio\}-4-[3-(1-methyl-1-silacyclopentyl)propyl]-1,2,4triazole (15b) in $34 \%$ yield correspondingly. 
Table 1. Synthesis of silacycloalkyl derivatives of heteroaromatic sulfides 8-14, 15a,b

\begin{tabular}{|c|c|c|c|}
\hline Thiol & Reaction time, $\mathbf{h}$ & Sulfide & Isolated yield, $\%$ \\
\hline $\mathbf{1}$ & 7 & $\mathbf{8}$ & $\mathbf{7 2}$ \\
\hline $\mathbf{2}$ & 7 & 9 & 46 \\
\hline 3 & 9 & $\mathbf{1 0}$ & 70 \\
\hline 3 & 5 & $\mathbf{1 1}$ & 70 \\
\hline $\mathbf{3}$ & 21 & $\mathbf{1 2}$ & 20 \\
\hline $\mathbf{5}$ & $\mathbf{8}$ & $\mathbf{1 3}$ & 26 \\
\hline $\mathbf{6}$ & 7 & $\mathbf{1 4}$ & 62 \\
\hline 7 & 3 & $\mathbf{1 5 a}$ & 32 \\
\hline & & $\mathbf{1 5 b}$ & 34 \\
\hline
\end{tabular}

The purity of the synthesized compounds was determined by HPLC ( $<1.5 \%$ of impurities). All substances were mobile oils therefore the elemental analysis was not performed.

The structures and spectral characteristics of synthesized substances are shown in Tables 2 and 3.

\section{PHARMACOLOGY}

CHOLESTEROL LEVEL LOWERING ACTIVITY

The Table 4 data show the serum lipid level at the end of the experiment. The high cholesterol in nutrition - Cholesterol group showed the marked increase in the total and LDL cholesterol in comparison to the intact control group. The HDL level in Cholesterol group did not differ from the Intact control group.

It has been found, that 2-\{[3-(1-methyl-1-silacyclopentyl)propyl]thio\}benzoxazole (9), 3-\{[3-(1methyl-1-silacyclopentyl)propyl]thio\}-4-[3-(1-methyl-1-silacyclopentyl)propyl]-1,2,4-triazole (15b), $\quad 1-$ methyl-2-\{[3-(1-methyl-1-silacyclopentyl)propyl]thio\}imidazole (8), and 2-\{[3-(1-methyl-1-silacyclopentyl)propyl]thio\} benzothiazole (10) produced a high antiatherosclerotic activity - protected against increase in serum LDL cholesterol level and atherosclerotic coefficient.

Benzoxazole and triazole derivatives 9 and 15b show the highest activity among all the investigated aliphatic [1] and silacyclic S-substituted heterocycles. A preliminary analysis of the structure-activity relationship for the cholesterol lowering action clearly indicates the strong influence of the silacyclic substituent position in the triazole ring. Compound 15a bearing a silacyclopentyl)propyl group in position 1 has slight effect on the cholesterol level $(\mathrm{K}=0.799)$. On contrary 4-N-substitution leads to a considerable increasing of activity: 3-\{[3-(1-methyl-1-silacyclopentyl)propyl]thio\}-4-[3-(1-methyl-1-silacyclopentyl)propyl]-1,2,4-triazole $(\mathbf{1 5 b})$ is 9 times more active $(\mathrm{K}=0.091)$ than the isomer 15a.

The silacyclic imidazole derivative 8 has better $K$ value $(K=0.111)$ than the corresponding trimethylsilylpropyl analogue $(\mathrm{K}=0.453)$ [1]. Moreover, compound 8 show the tendency to increase the HDL level. This fact indicates that this compound can possess an additional positive influence.

The activity of the silacyclic substituted benzothiazole $10(\mathrm{~K}=0.200)$ slightly differs from 2-(3trimethylsilylpropyl)thiobenzothiazole $(K=0.285)$ [1]. The insertion of a methylene group into the fivemembered silacyclopentane ring leads to decrease of activity (atherogenecity coefficient $K=0.200$ for 10 and $\mathrm{K}=\mathbf{0 . 4 0 1}$ for 11).

It is significant to note that benzoxazole derivative 9 is 3 times more active than benzothiazole derivative 10.

\section{VASODILATING ACTIVITY}

The vasodilating activity of the studied compounds in experiments in vivo is presented in the Table 4. In general the studied silacyclic compounds have a weak influence on vasodilatation even in a $50 \mu \mathrm{g} / \mathrm{mL}$ dose. It was found that imidazole derivative 8 exhibits the highest relaxation effect $(18 \%$ in $10 \mu \mathrm{g} / \mathrm{mL})$. It is more active than aliphatic silicon-containing analogues (12-13\%), but less active than dimethyl( $\beta$ triethylgermylethyl)silylmethyl substituted imidazole (22\%) [1]. 
Table $2 .{ }^{1} \mathrm{H}$ and ${ }^{13} \mathrm{C}$ NMR data of heteroaromatic sulfides

\begin{tabular}{|c|c|c|c|}
\hline \multirow[t]{2}{*}{ Cpd } & \multirow{2}{*}{${ }^{1} \mathrm{H}$ NMR, d (ppm, $\mathrm{CDCl}_{3}$ / HMDSO) } & \multicolumn{2}{|c|}{$\left.{ }^{13} \mathrm{C} \mathrm{NMR,} \mathrm{d} \mathrm{(ppm,} \mathrm{CDCl}_{3} / \mathrm{HMDSO}\right)$} \\
\hline & & Het & SR \\
\hline 8 & $\begin{array}{l}0.06\left(\mathrm{~s}, 3 \mathrm{H}, \mathrm{SiCH}_{3}\right), 0.51 \text { and } 0.72(\text { each } \mathrm{m}, 6 \mathrm{H}, \\
\mathrm{SiCH}), 1.51 \text { and } 1.67\left(\text { each } \mathrm{m}, 6 \mathrm{H}, \mathrm{CH}_{2}\right), 3.03 \\
\left(\mathrm{t}, 2 \mathrm{H}, \mathrm{J}=7.0 \mathrm{~Hz}, \mathrm{SCH}_{2}\right), 3.60(\mathrm{~s}, 3 \mathrm{H}, \mathrm{NCH} 3) \text {, } \\
7.91-8.05(\mathrm{~m}, 2 \mathrm{H} \text {, imidazole cycle })\end{array}$ & $\begin{array}{l}33.06\left(\mathrm{~N}-\mathrm{CH}_{3}\right) \\
121.83(\mathrm{C}-5), 129.06 \\
(\mathrm{C}-4), 141.85(\mathrm{C}-2)\end{array}$ & $\begin{array}{l}-3.00,11.51 \\
14.43,24.18 \\
27.16,37.62\end{array}$ \\
\hline 9 & $\begin{array}{l}0.08\left(\mathrm{~s}, 3 \mathrm{H}, \mathrm{SiCH}_{3}\right), 0.53 \text { and } 0.78(\text { each } \mathrm{m}, 6 \mathrm{H}, \\
\mathrm{SiCH}), 1.54 \text { and } 1.85\left(\text { each } \mathrm{m}, 6 \mathrm{H}, \mathrm{CH}_{2}\right), 3.31 \\
\left(\mathrm{t}, 2 \mathrm{H}, \mathrm{J}=7.2 \mathrm{~Hz}, \mathrm{SCH}_{2}\right), 7.2-7.6(\mathrm{~m}, 4 \mathrm{H}, \\
\text { benzoxazole cycle) }\end{array}$ & $\begin{array}{l}109.73(\mathrm{C}-6), 118.28 \\
(\mathrm{C}-5), 123.66(\mathrm{C}-7) \\
124.14(\mathrm{C}-4), 141.98 \\
(\mathrm{C}-7 \mathrm{a}), 151.74(\mathrm{C}-3 \mathrm{a}) \\
165.17(\mathrm{C}-2)\end{array}$ & $\begin{array}{l}-3.31,11.60 \\
14.64,24.61, \\
27.25,35.54\end{array}$ \\
\hline $\mathbf{1 0}$ & $\begin{array}{l}0.08\left(\mathrm{~s}, 3 \mathrm{H}, \mathrm{SiCH}_{3}\right), 0.53 \text { and } 0.78(\text { each } \mathrm{m}, 6 \mathrm{H}, \\
\mathrm{SiCH}), 1.54 \text { and } 1.85\left(\text { each } \mathrm{m}, 6 \mathrm{H}, \mathrm{CH}_{2}\right), 3.34 \\
\left(\mathrm{t}, 2 \mathrm{H}, \mathrm{J}=8.0 \mathrm{~Hz}, \mathrm{SCH}_{2}\right), 7.33 \text { and } 7.79(\mathrm{~m}, 4 \mathrm{H} \text {, } \\
\text { benzothiazole cycle })\end{array}$ & $\begin{array}{l}120.86(\mathrm{C}-6), 121.42 \\
(\mathrm{C}-5), 124.04(\mathrm{C}-7) \\
125.94(\mathrm{C}-4), 135.15 \\
(\mathrm{C}-7 \mathrm{a}), 153.35(\mathrm{C}-3 \mathrm{a}) \\
167.28(\mathrm{C}-2)\end{array}$ & $\begin{array}{l}-3.29,11.64 \\
14.78,24.58 \\
27.26,36.90\end{array}$ \\
\hline 11 & $\begin{array}{l}0.005\left(\mathrm{~s}, 3 \mathrm{H}, \mathrm{SiCH}_{3}\right), 0.65\left(\mathrm{~m}, 6 \mathrm{H}, \mathrm{SiCH}_{2}\right), 1.64 \\
\left(\mathrm{~m}, 8 \mathrm{H}, \mathrm{CH}_{2}\right), 3.35\left(\mathrm{t}, 2 \mathrm{H}, \mathrm{J}=7.4 \mathrm{~Hz}, \mathrm{SCH}_{2}\right) \\
7.25,7.74 \text { and } 7.87(\mathrm{~m}, 4 \mathrm{H} \text {, benzothiazole cycle })\end{array}$ & $\begin{array}{l}120.86(\mathrm{C}-6), 121.42 \\
(\mathrm{C}-5), 124.04(\mathrm{C}-7) \\
125.94(\mathrm{C}-4), 135.15 \\
(\mathrm{C}-7 \mathrm{a}), 153.35(\mathrm{C}-3 \mathrm{a}) \\
167.28(\mathrm{C}-2)\end{array}$ & $\begin{array}{l}-4.97,11.42 \\
12.73,12.78 \\
24.19,24.44 \\
30.10\end{array}$ \\
\hline 12 & $\begin{array}{l}0.13\left(\mathrm{~s}, 3 \mathrm{H}, \mathrm{SiCH}_{3}\right), 0.71\left(\mathrm{~m}, 6 \mathrm{H}, \mathrm{SiCH}_{2}\right), 1.71 \\
\left(\mathrm{~m}, 6 \mathrm{H}, \mathrm{CH}_{2}\right), 3.07\left(\mathrm{t}, 2 \mathrm{H}, \mathrm{J}=7.0 \mathrm{~Hz}, \mathrm{SCH}_{2}\right), \\
7.40,8.05 \text { and } 8.92(\mathrm{~m}, 6 \mathrm{H} \text {, quinoline cycle })\end{array}$ & $\begin{array}{l}121.45(\mathrm{C}-5), 123.56 \\
(\mathrm{C}-6), 123.75(\mathrm{C}-4) \\
126.44(\mathrm{C}-3), 128.15 \\
(\mathrm{C}-4 \mathrm{a}), 136.23(\mathrm{C}-7) \\
138.86(\mathrm{C}-8), 145.45 \\
(\mathrm{C}-8 \mathrm{a}), 149.04(\mathrm{C}-2)\end{array}$ & $\begin{array}{l}-3.35,11.55 \\
15.02,23.43 \\
27.19,34.38\end{array}$ \\
\hline 13 & $\begin{array}{l}\left.-0.01 \text { and } 0.00 \text { (both } \mathrm{s}, 6 \mathrm{H}, \mathrm{SiCH}_{3}\right), 0.57(\mathrm{~m}, \\
\left.12 \mathrm{H}, \mathrm{SiCH}_{2}\right), 1.2-1.8\left(\text { each } \mathrm{m}, 16 \mathrm{H}, \mathrm{CH}_{2}\right), 3.50 \\
\left(\mathrm{t}, 2 \mathrm{H}, \mathrm{J}=7.0 \mathrm{~Hz}, \mathrm{SCH}_{2}\right), 4.06(\mathrm{t}, 2 \mathrm{H}, \mathrm{J}=7.2 \mathrm{~Hz} \text {, } \\
\left.\mathrm{NCH}_{2}\right), 7.24 \text { and } 7.74(\mathrm{~m}, 5 \mathrm{H} \text {, indole cycle })\end{array}$ & $\begin{array}{l}109.68(\mathrm{C}-5), 119.59 \\
(\mathrm{C}-4), 119.76(\mathrm{C}-6) \\
120.52(\mathrm{C}-3), 121.97 \\
(\mathrm{C}-7), 130.13(\mathrm{C}-3 \mathrm{a}) \\
132.92(\mathrm{C}-7 \mathrm{a}), 136.53 \\
(\mathrm{C}-2)\end{array}$ & $\begin{array}{l}-4.97,-4.81 \\
11.42,12.58 \\
12.73,12.78 \\
13.12,24.19 \\
24.37,24.44 \\
24.73,30.01 \\
30.10,40.20 \\
\end{array}$ \\
\hline 14 & $\begin{array}{l}\left.0.08 \text { and } 0.08 \text { (both } \mathrm{s}, 6 \mathrm{H}, \mathrm{SiCH}_{3}\right), 0.51-0.83(\mathrm{~m}, \\
\left.12 \mathrm{H}, \mathrm{SiCH}_{2}\right), 1.54 \text { and } 1.82\left(\mathrm{each} \mathrm{m}, 12 \mathrm{H}, \mathrm{CH}_{2}\right) \text {, } \\
3.42\left(\mathrm{t}, 2 \mathrm{H}, \mathrm{J}=7.2 \mathrm{~Hz}, \mathrm{SCH}_{2}\right), 4.07(\mathrm{t}, 2 \mathrm{H}, \mathrm{J}= \\
\left.7.4 \mathrm{~Hz}, \mathrm{NCH}_{2}\right), 7.22 \text { and } 7.68(\mathrm{~m}, 4 \mathrm{H}, \\
\text { benzimidazole cycle) }\end{array}$ & $\begin{array}{l}108.59(\mathrm{C}-5), 118.13 \\
(\mathrm{C}-6), 121.48(\mathrm{C}-7) \\
121.51(\mathrm{C}-4), 136.12 \\
(\mathrm{C}-7 \mathrm{a}), 143.56(\mathrm{C}-3 \mathrm{a}) \\
152.07(\mathrm{C}-2)\end{array}$ & $\begin{array}{l}-3.33,-3.30 \\
11.52,11.61 \\
12.26,14.70 \\
24.35,24.61 \\
27.23,27.25 \\
35.86,46.95\end{array}$ \\
\hline 15a & $\begin{array}{l}0.06 \text { and } 0.07\left(\text { each } \mathrm{s}, 3 \mathrm{H}, \mathrm{SiCH}_{3}\right), 0.52 \text { and } 0.72 \\
\left(\text { each } \mathrm{m}, 6 \mathrm{H}, \mathrm{SiCH}_{2}\right), 1.53 \text { and } 1.78(\text { each } \mathrm{m}, 6 \mathrm{H}, \\
\left.\mathrm{CH}_{2}\right), 3.22\left(\mathrm{t}, 2 \mathrm{H}, \mathrm{J}=7.4 \mathrm{~Hz}, \mathrm{SCH}_{2}\right), 4.02(\mathrm{t}, 2 \mathrm{H}, \\
\left.\mathrm{J}=8.0 \mathrm{~Hz}, \mathrm{NCH}_{2}\right), 7.84(\mathrm{~s}, 1 \mathrm{H} \text {, triazole cycle })\end{array}$ & $\begin{array}{l}143.61(\mathrm{C}-5), 160.94 \\
(\mathrm{C}-2)\end{array}$ & $\begin{array}{l}-3.42,-3.31, \\
11.49,11.61, \\
11.95,14.58, \\
24.89,24.97, \\
27.21,27.24, \\
29.64,35.52, \\
52.79\end{array}$ \\
\hline
\end{tabular}




\begin{tabular}{|l|l|l|l|}
\hline $15 b$ & 0.05 and $0.07\left(\right.$ each $\left.\mathrm{s}, 3 \mathrm{H}, \mathrm{SiCH}_{3}\right), 0.52$ and 0.73 & $143.61(\mathrm{C}-5), 160.93$ & $-3.41,-3.29$, \\
& $\left(\right.$ each $\left.\mathrm{m}, 6 \mathrm{H}, \mathrm{SiCH}_{2}\right), 1.53$ and $1.78($ each $\mathrm{m}, 6 \mathrm{H}$, & $(\mathrm{C}-2)$ & $11.51,11.62$, \\
& $\left.\mathrm{CH}_{2}\right), 3.11\left(\mathrm{t}, 2 \mathrm{H}, \mathrm{J}=7.4 \mathrm{~Hz}, \mathrm{SCH}_{2}\right), 4.05(\mathrm{t}, 2 \mathrm{H}$, & & $11.97,14.60$, \\
& $\left.\mathrm{J}=8.0 \mathrm{~Hz}, \mathrm{NCH}_{2}\right), 7.96(\mathrm{~s}, 1 \mathrm{H}$, triazole cycle $)$ & & $24.89,24.98$, \\
& & & $27.22,27.25$, \\
& & & $35.52,52.79$ \\
\hline
\end{tabular}

Table 3. Mass spectra of heteroaromatic sulfides

\begin{tabular}{|c|c|}
\hline Sulfide & $\mathrm{m} / \mathrm{z}$ (relative int \\
\hline 8 & $\begin{array}{l}254\left(\mathrm{M}^{+}, 5\right), 225(40), 207(14), 197(29), 183(35), 170(34), 157(51), 141(31), 114(100), 99 \\
(23), 83(15), 71(31), 59(19), 43(34)\end{array}$ \\
\hline 9 & $\begin{array}{l}291\left(\mathrm{M}^{+}, 8\right), 276(18), 262(62), 249(49), 234(62), 220(100), 207(85), 194(48), 178(40), 167 \\
(13), 146(69), 133(57), 122(64), 99(89), 91(18), 83(15), 71(100), 59(50), 43(83)\end{array}$ \\
\hline 10 & $\begin{array}{l}307\left(\mathrm{M}^{+}, 11\right), 292\left(\mathrm{M}^{+}-\mathrm{Me}, 10\right), 280(13), 274(17), 265(14), 264(12), 263(18), 251(24), 250 \\
(39), 237(67), 236(51), 232(15), 223(18), 22(74), 209(29), 193(27), 179(12), 167(88), 162 \\
(100), 150(12), 149(62), 135(21), 122(14), 117(21), 109(12), 108(33), 102(17), 99(58), 97 \\
(52), 95(13), 75(28), 69(26), 59(44), 45(76), 43(72)\end{array}$ \\
\hline 11 & $\begin{array}{l}321(\mathrm{M}, 10), 278(100), 250(26), 237(30), 236(28), 223(21), 194(10), 167(40), 136(15), 113 \\
(15), 108(15), 85(46), 69(11), 59(24), 45(28), 43(27)\end{array}$ \\
\hline 12 & $\begin{array}{l}\left.301 \mathrm{MM}^{+}, 14\right), 286(14), 272(42), 254(51), 244(61), 230(27), 217(75), 202(81), 188(100), 174 \\
(65), 161(96), 142(40), 129(56), 116(24), 99(38), 89(20), 71(36), 59(24), 43(49)\end{array}$ \\
\hline 13 & $331\left(\mathrm{M}^{+}-\mathrm{SiMe}\left(\mathrm{CH}_{2}\right)_{5}, 10\right), 303(11), 149(45), 117(100), 113(90), 187(100), 85(82), 59(70)$ \\
\hline 14 & $\begin{array}{l}416\left(\mathrm{M}^{+}-\mathrm{Me}, 5\right), 415(14), 304(8), 261(31), 248(37), 234(13), 191(9), 150(25), 113(38), 85 \\
(100), 59(34), 43(22)\end{array}$ \\
\hline $\mathbf{1 5 a}$ & $\begin{array}{l}381\left(\mathrm{M}^{+}, 21\right), 380\left(\mathrm{M}^{+}-1,6\right), 351(18), 340(18), 324(10), 310(12), 298(18), 284(15), 282(14), \\
264(12), 242(42), 212(17), 210(14), 200(35), 186(17), 185(19), 184(17), 158(10), 121(15), \\
117(26), 99(100), 98(28), 97(58),, 85(15), 71(78), 59(35), 45(32), 43(33)\end{array}$ \\
\hline $\mathbf{1 5 b}$ & $\begin{array}{l}381\left(\mathrm{M}^{+}, 10\right), 366\left(\mathrm{M}^{+}-\mathrm{Me}, 10\right), 353(20), 325(60), 344(26), 339(16), 325(18), 324(32), 320 \\
(14), 305(16), 292(18), 282(16), 268(12), 242(15), 240(15), 226(16), 212(27), 200(15), 186 \\
(16), 170(15), 142(15), 128(10), 117(34), 99(100), 97(64), 85(20), 71(85), 59(32), 45(27),\end{array}$ \\
\hline
\end{tabular}

Table 4. Lipoprotein level lowering properties, vasodilating activity and acute toxicity of silacyclic derivatives of heteroaromatic sulfides

\begin{tabular}{|c|c|c|c|c|c|c|c|}
\hline \multirow[b]{2}{*}{$\mathbf{N}$} & \multicolumn{4}{|c|}{$\begin{array}{l}\text { The total cholesterol, } \\
\text { high and low density lipoprotein level } \\
\text { and the atherogenicity coefficient }(K)\end{array}$} & \multicolumn{2}{|c|}{ Vasodilating activity } & \multirow{2}{*}{$\begin{array}{c}\text { Acute toxicity } \\
\begin{array}{c}\mathbf{L D}_{\mathbf{5 0}} \\
(\mathrm{mg} / \mathrm{kg} \text {, i.p. })\end{array}\end{array}$} \\
\hline & $\begin{array}{c}\text { Total cholesterol } \\
\text { mg/dl }\end{array}$ & $\begin{array}{l}\text { HDL } \\
\mathbf{m g} / \mathbf{d l}\end{array}$ & $\begin{array}{c}\text { LDL } \\
\mathbf{m g} / \mathbf{d l}\end{array}$ & $\mathbf{K}$ & [ ], mM & \begin{tabular}{|c|}
$\begin{array}{c}\text { Relaxation } \\
\text { ("-" } \\
\text { contraction), } \\
\%\end{array}$ \\
\end{tabular} & \\
\hline Cholesterol & 124,7 & 70,7 & 54,0 & 0,825 & & & \\
\hline 8 & 99,9 & 90,5 & 9,5 & 0,111 & $\begin{array}{l}10 \\
50 \\
\end{array}$ & $\begin{array}{c}18 \\
30^{*}\end{array}$ & 375 \\
\hline 9 & 75,3 & 70,2 & 5,1 & 0,073 & $\begin{array}{l}10 \\
50\end{array}$ & $\begin{array}{l}12 \\
19\end{array}$ & $>800$ \\
\hline 10 & 99,7 & 82,9 & 16,7 & 0,200 & $\begin{array}{l}10 \\
50 \\
\end{array}$ & $\begin{array}{c}6 \\
15 \\
\end{array}$ & $>800$ \\
\hline 11 & 87,1 & 61,8 & 25,3 & 0,401 & $\begin{array}{l}10 \\
50\end{array}$ & $\begin{array}{l}-8 \\
19\end{array}$ & $>800$ \\
\hline 12 & 80,3 & 49,2 & 31,1 & 0,726 & $\begin{array}{l}10 \\
50 \\
\end{array}$ & $\begin{array}{c}-5 \\
12 \\
\end{array}$ & 580 \\
\hline 13 & 89,8 & 77,5 & 12,3 & 0,197 & $\begin{array}{l}10 \\
50 \\
\end{array}$ & $\begin{array}{c}8 \\
17 \\
\end{array}$ & $>600$ \\
\hline 14 & 88,7 & 55,2 & 33,5 & 0,643 & $\begin{array}{l}10 \\
50\end{array}$ & $\begin{array}{c}3 \\
15\end{array}$ & $>800$ \\
\hline 15a & 104,9 & 59,7 & 45,3 & 0,799 & $\begin{array}{l}10 \\
50\end{array}$ & $\begin{array}{c}9 \\
20\end{array}$ & $>800$ \\
\hline $15 b$ & 91,5 & 83,7 & 7,9 & 0,091 & 10 & 5 & 760 \\
\hline
\end{tabular}




\begin{tabular}{|c|c|c|c|c|c|c|c|}
\hline & & & & & 50 & 15 & \\
\hline $\begin{array}{c}\text { Intact } \\
\text { control }\end{array}$ & 69,0 & 64,9 & 4,1 & 0,065 & $\begin{array}{c}\text { Control/ } \\
\text { Solvent }\end{array}$ & 3 & \\
\hline
\end{tabular}

ACUTE TOXICITY

The studied compounds have basically a low acute toxicity (Table 4). Only imidazole sulfide 8 exhibits a medium level of toxicity $(375 \mathrm{mg} / \mathrm{kg})$.

\section{CONCLUSIONS}

A PTC method of synthesis of silacyclic derivatives of the N-, O- and S-heterocyclic sulfides was elaborated. Nine compounds were synthesized and isolated in the yields up to $70 \%$.

They were studied as serum cholesterol level lowering agents. It has been found, that silacyclopentylpropylthio-imidazole (8), -benzoxazole (9), -benzothiazole (10) and -1,2,4-triazole (15b) exhibited a high antiatherosclerotic activity. It protected against increase in serum LDL cholesterol level. A preliminary analysis of the structure-activity relationship for the cholesterol lowering action clearly indicates the strong influence of the silacyclic substituent position in the triazole ring. Imidazole derivative 8 has shown the tendency to increase the HDL level. This fact indicates that compound can possess an additional positive influence. The synthesized sulfides are low toxic compounds with weak vasodilating activity.

\section{ACKNOWLEDGEMENT}

The authors thank Latvian Council of Science for the financial support (Grant $\left.\mathrm{N}^{\circ} 166\right)$.

\section{REFERENCES}

1. K. Rubina, E. Abele, P. Arsenyan, R. Abele, M. Veveris, E. Lukevics, Metal Based Drugs, 8, 85 (2001).

2. A. Birch, R. Davies, L. Maclean, K. Robinson, J. Chem. Soc., Perkin Trans. 1, 387 (1994).

3. H. Hidaka, Jap. Patent, 04,330,057 (1992); Chem. Abstr., 119, 139132p (1993).

4. R.V. Davies, K. Robinson, PCT Int. Appl. WO Patent 9,102,724 (1991); Chem. Abstr., 115, 92095d (1991).

5. Tanabe Seiyaku Co., Ltd., Jap. Patent 8,130,991 (1981); Chem. Abstr., 95, 150717q (1981).

6. Tanabe Seiyaku Co., Ltd., Jap. Patent 57,134,469 (1982); Chem. Abstr., 98, 53714r (1983).

7. B.B. Lohray, V. Bhushan, A.S. Reddy, P.B. Rao, N.J. Reddy, P. Harikishore, N. Haritha, R.K. Vikramadityan, R. Chakrabarti, R. Rajagopalan, K. Katneni, J. Med. Chem., 42, 2569 (1999).

8. S. Raddatz, K.H. Mohrs, M. Matzke, R. Fruchtmann, A. Hatzelmann, R. Mueller-Peddinghaus, Ger. Patent 4,139,751 (1993); Chem. Abstr., 119, 271168j (1993).

9. N. Beier, J. Harting, R. Jonas, M. Klockow, I. Lues, G. Haeusler, J. Cardiovasc. Pharmacol., 18, 17 (1991).

10. R. Jonas, I. Lues, N. Beier, M. Klochow, K.O. Minck, Ger. Patent 4,041,074 (1992); Chem. Abstr., 117,151022u (1992).

11. R. Jonas, I. Lues, N. Beier, K.O. Minck, Eur. Patent 721,950 (1996); Chem. Abstr., 125, 168029q (1996).

12. M. Kidwai, N. Negi, P. Misra, J. Indian Chem. Soc., 77, 46 (2000).

13. M.P. Deninno, R.B. Ruggeri, R.T. Wester, Jap. Patent 2000 95,764 (2000); Chem. Abstr., 132, $251083 \mathrm{t}$ (2000).

14. Y. Nomura, S. Sakuma, S. Masui, PCT Int. Appl. WO Patent 96 12,719 (1996); Chem. Abstr., 125, $114599 \mathrm{~g}(1996)$.

15. M.S. Malamas, US Patent 5,037,831 (1991); Chem. Abstr., 115, 280004x (1991).

16. T. Aotsuka, T. Nishio, H. Hosono, Y. Nakamura, T. Matsui, H. Ishikawa, PCT Int. Appl. WO Patent 94 15,934 (1994); Chem. Abstr., 121, 280632z (1994).

17. H. Ishiguro, S. Shimada, M. Setani, Y. Yagi, N. Okane, Y. Saito, N. Takitsu, Jap. Patent 2000256,327 (2000); Chem. Abstr., 133, 222732u (2000).

18. T. Sohda, M. Makino, A. Baba, Eur. Patent 567,107 (1993); Chem. Abstr., 120, 217712q (1994).

19. M. Kato, S. Nishino, M. Hamano, H. Takasugi, PCT Int. Appl. WO Patent 93 10,097 (1993); Chem. Abstr., 120, 106772q (1994).

20. S.S.Pharmaceutical Co., Ltd., Jap. Patent 8,209,787 (1982); Chem. Abstr., 96, 217861y (1982).

21. R.M. Hindley, US Patent 5,002,953 (1991); Chem. Abstr., 115, 49719y (1991).

22. J. Frazee, C. Kaiser, L.I. Kruse, Eur. Patent 125,033 (1984); Chem. Abstr., 102, 132034y (1985).

23. S.S.Pharmaceutical Co., Ltd., Jap. Patent 6,075,487 (1985); Chem. Abstr., 103, 142015c (1985).

24. T. Fujita, K. Wada, M. Oguchi, H. Honma, T. Fujiwara, PCT Int. Appl. WO Patent 00 61,582 (2000); Chem. Abstr., 133, 296438z (2000). 
25. H. Sato, A. Nagai, K. Takayanagi, A. Tatsui, H. Yamada, K. Kojo, N. Narita, Jap. Patent 2001 19,678 (2001); Chem. Abstr., 134, 115957v (2001).

26. A.B. Brukshtus, V.N. Garaliene, A.R. Sirvidite, V.K. Daukshas, Khim.-Farm. Zh., 26, 50 (1992).

27. A.B. Brukshtus, V.N. Garaliene, A.R. Sirvidite, V.K. Daukshas, Khim.-Farm. Zh., 29, 30 (1995).

28. F. Kaiwa, M. Shoji, H. Domoto, H. Uchida, R. Yoshimoto, Jap. Patent 05,255,308 (1993); Chem. Abstr., 120, 245090d (1994).

29. T.P. Maduskuie, Jr., US Patent 5,179,117 (1993); Chem. Abstr., 118, 254933s (1993).

30. N.H. Dyson, J.O. Gardner, A. Prince, D.J. Kertesz, US Patent 5,208,331 (1993); Chem. Abstr., 119, 203411h (1993).

31. J.T. Billheimer, P.J. Gillies, C.A. Higley, T.P. Maduskuie, Jr., R.R. Wexler, PCT Int. Appl. WO Patent 91 18,885 (1991); Chem. Abstr., 116, 151761p (1992).

32. H. Iwabuchi, T. Fujiwara, T. Fujita, Jap. Patent 2001 97,954 (2001); Chem. Abstr., 134, 280842m (2001).

33. H. Yamaga, H. Yamaguchi, K. Maruta, M. Yasuchi, F. Hirota, J. Ichihara, Jap. Patent 2000109,467 (2000); Chem. Abstr., 132, 279218q (2000).

34. T. Fujita, K. Wada, M. Oguchi, H. Honma, T. Fujiwara, H. Iwabuchi, PCT Int. Appl. WO Patent 00 59,889 (2000); Chem. Abstr., 133, 281783j (2000).

35. H. Elokdah, T. Sulkowski, D. Cochran, M.-L. McKean, E. Quinet, Bioorg. Med. Chem. Lett., 10, 1791 (2000).

36. J.T. Billheimer, P.J. Gillies, R.R. Wexler, C.A. Higley, T.P. Maduskuie, Jr., Eur. Patent 372445 (1990); Chem. Abstr., 114, 6503k (1991).

37. R.G. Wilde, PCT Int. Appl. WO Patent 93 23,380 (1993); Chem. Abstr., 120, 217678h (1994).

38. R.G. Wilde, G. Richard, C.A. Higley, J.T. Billheimer, R.R. Wexler, PCT Int. Appl. WO Patent 93 23,381 (1993); Chem. Abstr. 120, 217676 f (1994).

39. K. Aoki, K. Aikawa, M. Kawakami, Y. Yan, PCT Int. Appl. WO Patent 01 04,110 (2001); Chem. Abstr., 134, 115956u (2001).

40. G. De Nanteuil, B. Portevin, A. Fradin, J. Bonnet, Actual. Chim., 48 (2000).

41. W.T. Ashton, M. Maccoss, L.L. Chang, P.K. Chakravarty, C.L. Cantone, W.J. Greenlee, A.A. Patchett, T.F. Walsh, Eur. Patent 409,332 (1991); Chem. Abstr., 115, 29337u (1991).

42. B.J. Teobald, PCT Int. Appl. WO Patent 01 19,826 (2001); Chem. Abstr., 134, 237500m (2001).

43. E.R. Larson, US Patent 5,175,179 (1992); Chem. Abstr., 118, 139845c (1993).

44. R. Steffan, A.A. Failli, PCT Int. Appl. WO Patent 99 11,618 (1999); Chem. Abstr., 130, 209600t (1999).

45. K. Luk, P.E. Mahaney, S.G. Mischke, PCT Int. Appl. WO Patent 00 35,906 (2000); Chem. Abstr., 133, 58710 n (2000).

46. N.J. Bach, S.E. Draheim, R.D. Dillard, E.D. Mihelich, J.S. Sawyer, D.W. Beight, M.L. Phillips, T. Suarez, D.J. Sall, J.A. Bastian, M.L. Denney, G.A. Hite, M.D. Kinnick, R.T. Vasileff, , M.J. Morin, Jr., H.-S. Lin, M.E. Richett, R.W. Harper, J.M. McGill III, B.A. Anderson, N.K. Harn, R.J. Loncharich, R.W. Schevitz, US Patent 6,177,440 (2001); Chem. Abstr., 134, 131518h (2001).

47. M.L. Jones, D. Gunn, J.H. Jones, M.C. Van Zandt, PCT Int. Appl. WO Patent 9950,268 (1999); Chem. Abstr., 131, 257442k (1999).

48. F. Watanabe, T. Takefumi, H. Tsuzuki, T. Shimamura, PCT Int. Appl. WO Patent 00 15,213 (2000); Chem. Abstr., 132, 236806n (2000).

49. J.W. Wilt, C.F. Dockus, J. Amer. Chem. Soc., 92, 5813 (1970).

50. N.S. Nametkin, K.S. Vdovin, K.S. Pushchevaya, V.I. Zavyalov, Izv. Akad. Nauk SSSR, Otd. Khim. Nauk, 1453 (1965).

Received: January 9, 2002 - Accepted: January 15, 2002 Accepted in publishable format: April 15, 2002 